\title{
Modelos de gestión de museos con inteligencia artificial
}

\section{Museum management models with artificial intelligence}

\author{
Diana María González Garza
}

México, formacion@evemuseos.com

\begin{abstract}
Resumen
Cada uno de nuestros museos es distinto, no solo en su clasificación o características sino en su contexto, historia, colecciones y modelos de gestión. Esta diversidad se traduce en distintas problemáticas en la dirección de cada uno de ellos y se intensifica al tratar de ofrecer soluciones genéricas basadas en líneas teóricas. Los nuevos modelos de negocio, basados en el análisis, interpretación de datos y definición de tendencias, han demostrado ser una auténtica referencia para organizaciones procedentes de muchos ámbitos, incluidas las empresas culturales y el sector turístico.

Organizar los recursos de forma eficiente ha sido una asignatura pendiente en muchas entidades, mas aun cuando lo exigen las transformaciones que el mundo nos impone haciendo frente a la incorporación de la tecnología y las herramientas digitales en todas sus formas. Los datos que podemos recopilar nos ofrecen grandes oportunidades, pero sumar esfuerzos con datos sobre búsquedas en temas de esparcimiento en general, perfiles demográficos de la comunidad a la que pertenece el museo, etcétera, nos llevan a la construcción de un modelos hechos a la medida y que responden a públicos especializados $y$ diversos, identificando al mismo tiempo conexiones potentes de información y observando aquellas que parecieran débiles a simple vista para crear nuevas oportunidades que sean atractivas a nuestras comunidades.

El sector turístico por ejemplo, ha adoptado muchas formas de inteligencia artificial, una de ellas llega a ofrecer su registro de actividades complementarias a sus viajeros, pero hay detrás de ello información valiosa, como son sus gustos, horarios libres, cantidad de personas que viajan con cada usuario, etc. Estas lecturas nos permitirán ofrecer al público, desde nuestros museos, alternativas distintas pensadas y ajustadas a sus necesidades y gustos personales, y con ello gestionar los propios recursos del museo de forma coherente y disruptiva.
\end{abstract}

Palabras clave: Museos; Inteligencia artificial; Gestión de museos; Modelos organizacionales; Modelos de negocio. 


\begin{abstract}
Each one of our museums is different, not because it's classification or it's characteristics, but because of its context, history, collections and management models. This variety translates to different problems in their management and intensifies when you offer generic solutions only based on theory. The new business models, based on analysis, data interpretation and trend determination, have shown being a true reference to organizations from different fields, including cultural companies and the tourism sector.

Organizing resources in an efficient way has been a pending subject in many entities, even more when the changes that the world forces on us demands incorporating technology and digital tools in all their forms. The data you can collect offers big opportunities, but if you join forces with data in general, demographic profiles of the community which the museum belongs to, etcetera, it gives you a custom-made model that responds to specialized and diverse audiences, at the same time identifying strong information connections and analyzing those that seem weak to create new opportunities that are attractive to our communities.

The tourism sector for example, has adopted many forms of artificial intelligence, one of them is when they offer you to sign up for complementary activities, there is a lot of valuable information behind it, like their interests, schedule, number of people traveling with each user, etcetera. This information will allow us to offer the public different options adjusted to their necessities and personal interests, and with that, manage the museum's resources in a consistent and disruptive way.
\end{abstract}

Keywords: Museums. Artificial intelligence. Museum management. Organizational models, Business models 
"La inteligencia artificial se ha infiltrado en nuestras vidas, a menudo sin que nos demos cuenta, a diario. Y lo usamos para realizar tareas absolutamente fundamentales "

Sara Boutall, 2018. MuseumNext Londres

\section{Introducción}

Desarrollar un pensamiento a la vez crítico y creativo en torno a las funciones y actividades que se llevan a cabo en nuestros museos es en ocasiones abrumador, sin embargo, podría ser más accesible desarrollar este proyecto si tomamos un poco de tiempo para reconstruir de forma muy breve la evolución que estas instituciones han tenido a lo largo del tiempo y tomarlo como punto de partida para establecer algunas pautas que sirvan de guía en el trabajo del museo, considerando las actuales necesidades de sus audiencias o públicos y aprovechando a la vez los beneficios que la tecnología digital ofrece.

En el comienzo de la historia de los museos, desarrollada entre los años de 605 - 562 A.C en el palacio del rey Nabucodonosor II, en la Antigua Babilonia, se buscaba perpetuar la memoria y el coleccionismo puro, iniciando así la experiencia asociada con la recogida, conservación y exposición de objetos o piezas valiosas, este conocimiento se ha ido desenvolviendo y transformando conforme lo ha hecho la sociedad misma a través de las diferentes épocas y las exigencias que cada cambio de período suponen, transitando de forma constante por los acontecimientos de la sociedad hasta el día de hoy. (León, Aurora, 2010. El Museo: Teoría, praxis y utopía). Tomando como referencia el concepto de museo universal y actualmente aceptado, propuesto por el ICOM (International Council of Museums) en cuyos estatutos establece:

"Son museos las instituciones de carácter permanente, sin fines de lucro, al servicio de la sociedad, que adquieren, conservan, investigan, comunican y exhiben para fines de estudio, educación y contemplación, conjuntos y colecciones de valor histórico, artístico, científico y técnico o de cualquier otra naturaleza cultural."

$\mathrm{Y}$ las funciones que del mismo concepto se desprenden:

- Conservadora.

- Investigadora, para el estudio de los objetos conservados.

- Educativa.

- Difusora, por medio de la exposición, principalmente.

- Y de deleite o emocional. 
Comprendemos que de los puntos anteriores se derivan cada una de las misiones, objetivos, estrategias, planes, y actividades cotidianas en la vida de nuestros museos y con ello se escribe la agenda de quienes formamos parte de ese ecosistema, atendiendo de forma prioritaria dichas funciones como eje central de nuestra propia actividad cotidiana.

Algunas de las no pocas inquietudes que los equipos de trabajo del museo debe resolver tienen que ver con el perfil del público, inquietudes como: las que EVE Museos e Innovación plantea : ¿quiénes son?, ¿de dónde vendrán?, ¿a dónde irán después de la visita?, ¿qué les habrá traído hasta aquí?, ¿estarán pasándolo bien?, ¿estarán aprendiendo algo?... han sido planteadas una y otra vez por museólogos, museógrafos y equipos de trabajo de los museos en general. Estas instituciones se preocupan cada vez más por conocer al público que reciben y reflexionan sobre cómo saber más de esas personas, determinando qué datos son precisos y valiosos, cuales son aquellos que está dispuestos a proporcionarnos y cuales de ellos podemos "detectar" con ayuda de instrumentos digitales, todo ello para diseñar estrategias relacionadas capaces de mejorar la experiencia de los visitantes. Se trata de proponer y llevar a cabo acciones que sean atractivas y puedan captar el mayor público al museo.

Estas reflexiones indican una clara preocupación por "recabar información" de los visitantes, por tener conocimiento de sus perfíleles, gustos, aficiones, preocupaciones, lugar de residencia y una larga lista de detalles cada vez más específicos y complejos. No es nuevo que los museos y centros culturales estén buscando la manera de ser parte activa en la vida de sus comunidades y visitantes, y han hecho al día de hoy, un enorme esfuerzo por obtenerlos.

Transformar dicha información en escenarios posibles no es sencillo, hace falta más que solo contar con ellos, es necesario un equipo multidisciplinario capaz de recrear modelos matemáticos en aquello que representen tendencias o inclinaciones de la audiencia, del mercado, la economía o situación en general que en el mundo ocurre. Es necesario construir una arquitectura que administre dichos datos e identificar el posible valor a través del tiempo de las conexiones valiosas entre ellos. En este contexto se hace imprescindible contar una planeación óptima de las actividades tomando en cuenta el potencial de los datos obtenidos, sus interpelaciones y sus limitaciones. Es evidente la importancia de alinear las estrategias digitales desde la Dirección de cada museo, con la misión, objetivos y actividades del mismo para lograr el equilibrio entre el uso de la tecnología al servicio de la comunidad interna y externa, sin dejar de mantener su existencia y atracción física como instituciones al servicio público.

\section{Modelos de Gestión de museos y desarrollo}

El modelo de desarrollo organizacional en el ámbito de la cultura y las artes se lleva a cabo respondiendo a la transformación digital y a las demandas (también nuevas) de la política cultural. En cualquier caso, el desarrollo organizacional es un proceso complejo, que incluye estrategias de formación y actualización para mejorar las capacidades de quienes conforman los equipos de trabajo en organizaciones e instituciones, y que pretendan operar con éxito 
durante un largo período de tiempo. Ayudar a la organización a adaptarse a los cambios, y a responder con agilidad es el objetivo primordial de la disciplina denominada desarrollo organizacional y será la base para adaptarse a los cambios esperados e inesperados en el entorno presente y futuro, ya que son nuestros propios equipos quienes tienen la responsabilidad de llevar a buen puerto la misión del museo.

Tres modelos organizacionales han sido históricamente considerados para agrupar eficientemente el personal del museo, y destacan las diferentes formas en que la administración del museo puede facilitar el trabajo en equipo:

- Pirámide jerárquica.

- Organización matricial.

- Fuerzas de trabajo.

Estos tres modelos no son excluyentes y pueden actuar de forma combinada, cuando así se considere necesario. Numerosas autoridades, líderes mundiales y expertos en museos han participado en el desarrollo de teorías sobre la economía empresarial y la administración de museos. Este es el caso del analista y planificador de museos John W. Jacobsen. Jacobsen 2016, proporciona tanto los fundamentos teóricos como la pragmática operativa para medir el impacto y el rendimiento de cualquier museo, la conclusión ha sido que el conocimiento sobre la medición proporciona a sus profesionales un proceso claro y muy abierto. Todo ello mejorará el valor y el rendimiento de nuestros museos mediante la selección de indicadores capaces de controlar si se están cumpliendo los valores públicos, privados, personales e institucionales deseados. Este marco reconoce que cada museo necesita decidir sobre sus propios propósitos y prioridades, y con ello seleccionar aquellos indicadores que sean relevantes para cumplir dichos objetivos.

En los últimos años hemos visto como Internet ha transformado el modelo de negocios y la forma de organizar las entidades en todos los sectores, desde los medios de comunicación, la transportación, el sector financiero, turismo, y cualquier industria que podamos imaginar. El sector cultural no es la excepción, y la transformación también se manifiesta en el modelo de gestión que se sigue o debiera seguirse y los tres modelos que históricamente hemos seguido resultan ahora insuficientes. La transición es obligatoria desde la base de la estructura, el mundo de las organizaciones responde a los comportamientos de sus mercados o públicos, en el caso de los museos lo denominaremos tendencias, para desarrollar soluciones pertinentes en cada caso. De acuerdo a la firma dosdoce.com para Cedro. (Nuevos modelos de negocio en la era digital, 2014).

Es difícil tener claro cuál es el modelo que perdurará o cuál será el más sostenible en cada caso, no hay una respuesta sencilla y menos una ruta clara a seguir. Habrá muchos modelos, panoramas, escenarios que diseñar, desarrollar y evaluar, y sin duda muchos podrán cohabitar entre sí. El éxito de cada modelo dependerá de cada museo, de su capacidad para adaptarse y crear a partir de eso un catálogo de variables y de posibilidades que generen la mezcla de soluciones adecuadas para su propio museo con una mentalidad flexible, adaptable y 
resilente, a sabiendas que innovar conlleva perder el miedo a cometer errores y superarlos. En muchas ocasiones vamos a encontrar modelos mixtos, fruto de la conjugación de varios de ellos ya que es difícil encuadrar estrictamente uno u otro modelo, y como en el caso de los modelos organizacionales clásicos, no son excluyentes; la nueva economía se define por las relaciones que el internet nos facilita y es obligatorio para sobrevivir encontrar cuales son las relaciones optimas en cada caso. A continuación enumeramos algunas de las propuestas en los nuevos modelos de negocio señalados por esta misma firma, modelos de negocio en la era digital, modelos que podremos complementar sin duda, pero que proponemos como punto de partida:

- Contenidos fraccionados. Micropagos.

- Pago por consumo streaming. Pay per View.

- Suscripciones, membresias y premium o freemium.

- Publicidad inserta.

- Acceso abierto.

- Peer to peer (P2P, “de igual a igual”).

- Paga lo que quieres. (Pay what you want).

- Bundle (paquetes).

- Crowdfunding / crowdsourcing. (Micromecenazgo o financiación de proyectos).

- Gamificación.

- Venta directa.

- Del espacio de venta físico al digital.

- Big data como clave del modelo de negocio digital.

- Modelos de convergencia. Del todo gratis a la diversificación.

- Consumidores activos: prosumidor, y contenidos generados por usuarios (UGC).

Cada uno de estos modelos resulta de la búsqueda constante por ofrecer a los usuarios mejores experiencias, de formas flexibles, abiertas y probablemente hasta co-diseñadas con el propio usuario. Es importante para elegir el mix de modelos que sea exitoso en cada organización cultural o museo, conocer al público, su perfil, necesidades, y reflexionar profundamente cuál es la estrategia que mejores resultados brindará al museo, tema del que hablamos con anterioridad y que retomaremos un poco más adelante.

La disciplina conocida como «previsión estratégica» es una forma de pensar, aplicando una metodología que nos ayudará a manejar la incertidumbre y a preparar respuestas flexibles y adaptables. Intenta dar respuesta a planteamientos como: 
- ¿Cuáles son las fuerzas de cambio más importantes que darán forma al sector de los museos durante el próximo año y década?

- ¿A qué desafíos se enfrenta el campo de los museos en su conjunto?

- ¿Cuál es el perfil de riesgo de un museo como los nuestros: qué tendencias y disrupciones podrían amenazar su supervivencia?

- ¿Qué estrategias podrían contribuir al éxito de los museos en los próximos años, independientemente de cómo se desarrolle el futuro?

Una de las lamentaciones pandémicas que escuchamos con más frecuencia fue: «inadie lo vio venir!» Pero más bien fue que no pusimos atención en los hechos. Los expertos habían previsto que una pandemia mundial no solo era probable, sino inevitable en algún momento. Uno de los primeros ejercicios de pronóstico dirigidos por el Centro para el Futuro de los Museos, hace 12 años, reclutó a una serie de museos para participar en una simulación creada por el Instituto para el Futuro sobre una pandemia global. De hecho, las pandemias no son las únicas perturbaciones «imprevistas»: que son predecibles. Del mismo modo, en algún momento del próximo siglo habrá un gran terremoto a lo largo de una o más de las fallas en la costa oeste de Estados Unidos. Existen otros muchos escenarios que son predecibles. Estas disrupciones se superpondrán a un panorama de cambio existente: tendencias que han estado reconfigurado la cultura, la economía, la tecnología, los sistemas políticos y nuestro medio ambiente, por mencionar algunos.

Pero la realidad nos indica que pocos son los museos preparados para adaptarse al alcance y ritmo de todas esas transformaciones. Cuando el trabajo de todos y cada uno es necesariamente a largo plazo: preservar el patrimonio cultural y científico para la próxima generación, servir a su comunidad y ayudarla a prosperar. Por ello, los museos deben dedicar tiempo a planificar futuros inciertos, regresar a la práctica de revisar el concepto del que partimos "de carácter permanente, al servicio de la comunidad, que adquiere, conserva....." O todo el esfuerzo y enorme trabajo que hacen día a día está en riesgo.

Los museos, desde su gestión deben mejorar su planificación como base del proceso de toma de decisiones con «previsión estratégica», una mentalidad y una metodología que ayude a las organizaciones a abordar la incertidumbre y preparar respuestas flexibles y adaptables. En lugar de utilizar un plan que los guíe a través de un paisaje familiar hacia un destino conocido, los museos deben aprender a seguir adelante aún sin saber lo que les espera, esquivando y resolviendo en el peor de los casos, y medida que surjan nuevos obstáculos, recalibrando constantemente su curso hacia un futuro mejor. Y así organizar a sus equipos de la mejor forma, o reorganizar si es la respuesta adecuada.

Un ejemplo es la dependencia de muchos museos del turismo local, nacional e internacional, todos ellos cerrados por la pandemia. Pero el turismo también podría haber sido interrumpido por otros motivos: sanciones políticas o económicas, colapso del transporte público, aumento de los precios de la gasolina, etcétera. Al identificar el turismo como una vulnerabilidad crítica, los museos diseñarán estrategias que se tornarán en acciones a implementar cuando 
dicho turismo se vea dañado. Es necesario incorporar herramientas de planificación que sintetizan innumerables futuros posibles en un número manejable de alternativas que cubren diversos resultados para las fuerzas de cambio más importantes.

\section{Inteligencia Artificial (IA). Compromiso con los visitantes}

Cuando se trata de museos y en general de centros culturales, la Inteligencia Artificial (IA por sus siglas en inglés) se puede incorporar en todo el hábitat que lo conforma, desde la experiencia del visitante hasta lo que ocurre detrás de escena; y la tecnología puede participar de ello, y se ha incorporado en muchas formas, (Lauren Styx. Museumnext, How are museums using artificial intelligence, and is a AI the future of museums?)., Un ejemplo es Museums on the Web, quienes han hecho el esfuerzo de observar nuevas prácticas respecto al uso de la tecnología, como lo hace el Netherlands Institute for Sound and Vision, (Instituto Holandés de Sonido y Visión), utilizando laboratorios como instrumentos estructurados pero flexibles para experimentar con diferentes soluciones que se requieren para la realización de una nueva experiencia de visitante en una etapa temprana. "Nos permiten evaluar el desempeño funcional, la experiencia del usuario y los aspectos prácticos involucrados en la implementación de diferentes soluciones tecnológicas para los muchos desafíos involucrados con nuestra ambiciosa visión para el nuevo museo. Los laboratorios se llevan a cabo en parte "en la naturaleza" en el piso del museo, con sujetos de prueba que representan al nuevo visitante potencial del museo. En los próximos años, estos laboratorios nos permitirán fracasar temprano, tomar decisiones informadas y desarrollar conocimiento interno sobre aspectos clave de nuestra nueva experiencia en el museo y los mecanismos necesarios para hacer realidad nuestra visión."

Dicho planteamiento, redefine la propuesta del nuevo museo, aquello que Hegley (2016) llama el "museo ágil". Este tema trata sobre el posible despliegue de agentes como robots, o asistentes virtuales conversacionales para comunicarse con los visitantes pudiendo incorporar esta infraestructura en diferentes niveles y puntos de contacto en el recorrido del visitante como puede ser:

- Bienvenidas, introducción, información y despedidas.

- Cuestionarios y comentarios.

- Guías del museo, ubicación de distintas áreas.

- Narración del recorrido.

- Contextualización, de las obras.

- Preguntas y respuestas.

- Acceso al archivo o profundizar en información relevante.

Esta experiencia concentra una gran cantidad en la recogida de datos, desde temas no complejos como el discurso entre los visitantes o el idioma, las palabras más usadas o 
buscadas, preguntas frecuentes, hasta situaciones mas profundas, como podrían ser los gestos, entrada o trayecto físico que siguen, tiempo del recorrido, el registro visual, ondas cerebrales, reconocimiento facial, biométrica. Aunado a ello, contamos con la información generada por los dispositivos personales, los dispositivos de apoyo en las experiencias inmersivas, y los diseños responsivos de interacción multimodal en distintos niveles y presentaciones, todos y cada uno agilizan la recogida de todo tipo de información, aquella que es inmediatamente reconocida como relevante y aquella que debiera parecer poco importante y que sin embargo su uso marcará una diferencia en los resultados y las estrategias. Toda la información reunida proporciona una gran oportunidad para definir posibles escenarios optimizando los datos que se obtiene. El museo necesitará determinar la forma más rentable de interactuar con los visitantes y evaluar su estrategia continuamente para comprobar qué canales son los más adecuados a partir del cruce de estos datos, de como se utilizan, de que relaciones entre ellos aparentan ser menos fuertes y que sin embargo brindan enfoques distintos.

Los museos hoy se enfrentan con problemas de supervivencia acrecentados por la pandemia de COVID-19, sin embargo la problemática tiene origen años atrás; por ejemplo en el año 2016 se presenta el NMC Horizon Report Museum Edition, que recoge una serie de 18 tendencias en el futuro de los museos. La preocupación por mantenerse actualizados y a la vanguardia para satisfacer las necesidades de la audiencia continua, ahora con un componente que no esperamos: Pandemia, y la que hace el momento oportuno para transformar los retos en oportunidades de cambio, como lo señalamos inicialmente: creciendo y progresando conforme lo ha hecho la sociedad misma y las exigencias que cada cambio de época suponen transitando de forma constante por los devenires de la sociedad y sus particulares situaciones. Si no se trabaja bajo este enfoque, realmente estaremos perdiendo muchas oportunidades. Las 18 tendencias presentadas en el año 2016 se enumeran a continuación, y siguen tan vigentes como hace 5 años, o probablemente se presentan hoy como hechos inevitables y se exige su incorporación en el mundo cultural actual.

1. Colaboración entre Instituciones.

2. Nuevos roles para profesionales del museo.

3. Análisis de datos para las operaciones del museo.

4. Personalización.

5. Contenido móvil y entrega.

6. Experiencias participativas.

7. Desarrollo de estrategias digitales efectivas.

8. Mejora de la alfabetización digital de los profesionales del museo.

9. Mejora de la accesibilidad para las poblaciones con discapacidad.

10. Medición del impacto de las nuevas tecnologías. 
11.Gestión de la obsolescencia del conocimiento.

12. Preocupaciones sobre la privacidad.

13.Tecnologías de humanidades digitales.

14. Makerspaces.

15. Inteligencia de localización.

16. Realidad virtual.

17. Visualización de información.

18. Objetos en red.

\section{Gestión y modelos digitales para la toma de decisiones}

Un modelo es una representación de la realidad que sirve para resumir la complejidad y para transmitir conocimientos o experiencias que luego deben ser adaptados a otra realidad. La tecnología permite obtener respuestas más rápidamente, cometer menos errores $\mathrm{y}$, a veces, curiosamente, comprender mejor a nuestros semejantes. (Nextmuseum). La tecnología y sus usos se han desarrollado aceleradamente y los museos han ido adoptando a su gestión aquello que consideran útil. Sin embargo, su utilidad se refleja en la medida que facilitan la explicación de una realidad compleja y facilitan la transmisión de experiencias de otros casos y en la implementación de procesos, no porque ofrezcan soluciones universales a los problemas específicos de cada organización, sino porque ayudan a visualizar nuevas formas de trabajo, distintas perspectivas y oportunidades que favorecen el crecimiento de la organización. Por ello surgen continuamente nuevos modelos y variaciones. En los modelos de gestión, lo que cuenta es lo que se hace con ellos, las decisiones que a partir de esta información se tome, más que el modelo en sí.

El auge que ha adquirido la inteligencia artificial ha puesto en evidencia que las máquinas están aquí para asumir numerosas tareas que hace apenas poco tiempo eran reservadas a los humanos. Su presencia trasciende laboratorios y centros de pruebas, y adquiere una dimensión real en la operación de las organizaciones. El potencial de la informática para automatizar tareas aumenta, es momento de encontrar las mejores opciones de colaboración entre hombre y máquina. Finalmente, en lugar de orientar la innovación tecnológica exclusivamente a una sola área, es oportuno plantearnos cómo crear valor para los clientes actuales y como identificar a los clientes potenciales para brindarles nuevos formatos de comunicación, además de generar significado para los equipos de trabajo; todo a partir de la información que ahora leemos como escenario.

Una posición más humanizada que la inspirada en la ciencia de los años 50, cuando arrancan los primeros trabajos sobre inteligencia artificial, cuyo enfoque reposaba en gran medida en poner frente a frente las capacidades de los seres humanos y las de las máquinas. Por el contrario, en los últimos tiempos -y con especial incidencia desde el comienzo de la crisis 
sanitaria-, la aproximación que prima es poner al ser humano en el centro de la innovación y de la adopción de tecnología.

Recientemente, los museos que utilizan la tecnología de inteligencia artificial, lo hacen para atraer al público y personalizar las experiencias de los visitantes. Antes de realizar más investigaciones, es importante comprender cuáles son las tecnologías de IA empleadas, ¿Cómo se implementan en los museos? ¿Y hay desafíos o problemas? Un resumen de las subcategorías de IA que los museos exploran con mas frecuencia, así como una breve descripción de las mejores prácticas y las actividades en las que se implementa la IA, además de los desafíos que esta aplicación de la tecnología podrían traer se mencionan a continuación.

Entre todas las subcategorías de IA, las tres secciones mencionadas con más frecuencia son: Chatbot, Aprendizaje Automático / Red Neuronal y Aprendizaje Profundo. La primera subcategoría es Chatbot, un robot de software que interactúa con humanos y se conecta con las audiencias personalizando el recorrido e interactuando con ellas a través de un servicio inteligente dirigido al público.

En segundo lugar, el aprendizaje automático, la tecnología podría aprender, categorizar y hacer predicciones sobre datos existentes. El análisis de datos podría utilizarse en el reconocimiento facial (estilo, color, textura, reconocimiento de objetos de las obras de arte), seguimiento geográfico en las redes sociales y seguimiento de asistencia para mostrar la preferencia del público por la estrategia de exhibición futura, entre otras muchas cosas.

Por último, pero no menos importante, basado en el aprendizaje automático, la red neuronal / aprendizaje profundo también está cambiando las experiencias del museo. Neural Network / Deep Learning son sistemas informáticos que crean nuevos datos que sé parecen a un conjunto de datos existente que está modelado libremente en el cerebro humano. Esta tecnología se puede utilizar en la investigación de archivos en museos. Por ejemplo, un sistema de aprendizaje profundo puede reconocer documentos antiguos escritos a mano en latín y convertirlos en digitales basándose en su gigantesco banco de memoria. Además, la red neuronal también puede cambiar las formas en que los artistas crean arte. Las Redes Adversarias Generativas (GAN) podrían ser un gran ejemplo y ya se utilizan en la exposición "Memories of Passerby I" y un prototipo de "Mapas genéricos" hecho por Met, Microsoft y MIT. Lingxi, May 2020. Audience Engagement, Exhibitions.

La necesidad de un nuevo contrato o pacto social digital es una cuestión propuesta abiertamente, como es que cada museo o centro cultural conjuga las herramientas dependiendo de misiones, objetivos, habilidades es tarea de cada uno de los equipos y sus administraciones. Cada institución deberá ser capaz de identificar y seleccionar los indicadores acordes a sus planeaciones estratégicas, y de señalar rutas de lectura distan tas que den como resultado nuevas propuestas.

De una forma u otra, todos los trabajos se verán afectados por la implantación de estas tecnologías. La innovación requerirá la relocalización de recursos y la recualificación de los 
trabajadores, no solamente en temas técnicos, sino en habilidades tan "humanas" como pueden ser la creatividad, la comunicación, y la capacidad para juzgar y tomar decisiones.

El Foro Económico Mundial reconoce que para el año 2022 el 50\% de las empresas prevé que la automatización les habrá llevado a reducir fuerza de trabajo basada en los perfiles actuales (más bien, los que existían en 2018, cuando se llevó a cabo la encuesta). La buena noticia es que el $38 \%$ de las mismas esperan que este proceso lleve a la plantilla a asumir funciones más productivas dentro de la empresa, y más de la cuarta parte confía en que la introducción de tecnología llevará a crear nuevos perfiles profesionales.

\section{Conclusiones}

Se espera una creciente necesidad de perfiles asociados a las tecnologías de vanguardia . Una encuesta realizada por MindEdge a trabajadores estadounidenses señala que el $76 \%$ de aquellos en cuyas compañías ha integrado la inteligencia artificial considera que su trabajo ha sido simplificado, y el 69\% declara que ha mejorado el clima laboral. Colaboración entre humanos y máquinas.

A pesar de que una visión de corto plazo puede llevar a los a sustituir mano de obra por programación, hay estudios que demuestran que, a la larga, se obtienen mejores resultados combinando inteligencia artificial y trabajo humano. Wilson y Daugherty de Accenture llevaron a cabo una investigación con 1,500 empresas en la que se pone en evidencia que el trabajador y la máquina se refuerzan y complementan mutuamente. Se trata de combinar las fortalezas humanas -como la capacidad de liderazgo, de trabajo en equipo, la creatividad y las habilidades sociales-, con las ventajas que ofrecen los algoritmos: la velocidad y escalabilidad en el trabajo cuantitativo con inmensas cantidades de datos. La colaboración entre personas y máquinas requiere de un rediseño de los procesos del negocio para que cada parte puede "sacar lo mejor" de la otra.

De esta forma y de acuerdo con el estudio expuesto, los trabajadores deben llevar a cabo tres funciones dentro de este trabajo en equipo: entrenar a los sistemas para que realicen determinadas tareas (el aprendizaje automático requiere alimentar a los algoritmos de grandes cantidades de datos para que aprendan a hacer lo que queremos que hagan); igualmente, deben poder explicar los resultados que ofrece la inteligencia artificial a aquellos no familiarizados con ella, especialmente si son conclusiones poco intuitivas o controvertidas; por último, los "compañeros humanos" de las máquinas deben mantenerlas, es decir, velar porque estas funcionen de forma responsable, por ejemplo, evitando que un autómata pueda dañar a las personas, o que un algoritmo ofrezca resultados sesgados que puedan conducir a una situación de discriminación.

Las conclusiones que presenta la consultora Deloitte sobre este tema son similares: los sistemas inteligentes deben aumentar y amplificar las capacidades humanas. Los expertos proponen reimaginar el trabajo, en vez de como un conjunto de tareas organizadas en un proceso predefinido, como un esfuerzo colaborativo en el que los humanos definen un 
problema, las máquinas ayudan a hallar las soluciones, y los humanos verifican la validez de esas soluciones además de llevar su implemantación. La automatización no debería justificar reducciones de personal, sino que tendría que liberar a los empleados de las tareas más monótonas y repetitivas, para que puedan llevar a cabo funciones más creativas. Deberá sin dudar a tomar decisiones mas acertadas y ponerlas en marcha.

Es el momento ideal para que los museos puedan incorporar nuevas opciones en sus propuestas que brinden resultados acordes a las nuevas necesidades, es momento de plantearse cómo incorporar en su labor cotidiana las herramientas digitales? Qué es aquello que requieren? Ya tienen un plan en el que se describa dicho transito?. Las nuevas herramientas de trabajo incorporan el uso de inteligencia artificial y proporcionan nueva información para tomar mejores decisiones de forma más ágil, además de la gestión coherente y oportuna de los recursos en función de datos, conexiones y nuevas relaciones.

Si el futuro es el destino, la tecnología es el camino directo, y esta suele ser la principal responsable de los grandes cambios que hemos vivido en los últimos siglos: desde la imprenta de Gutenberg hasta el cable de teléfono o, por supuesto, Internet. Cada invento nos ha permitido explorar nuevos caminos y, bajo ciertas circunstancias nos da la oportunidad de cambiar el mundo tal y como lo conocíamos para proponer un mundo mejor. Hoy en día, la tecnología evoluciona a un ritmo acelerado lo que desencadena, por ejemplo, la metamorfosis repentina de sectores como el del turismo. Lo que antes era ciencia ficción, está a punto de hacerse realidad en el presente: el hyperloop, los coches voladores o el turismo espacial, todos son ya tangibles. Este fenómeno es una oportunidad y sobre todo una herramienta para llegar a nuestro destino: el futuro próximo, y de la mano de las tecnologías aplicadas conseguiremos llegar mas lejos.

Sin duda cada museo es distinto, no solo por sus características sino también por su contexto. La manifestación de urgencia referente al cambio y a la utilización de tecnología digital se ha hecho presente y con sentido de dinamismo no solamente para recabar datos, sino cruzarlos y dar lecturas oportunas y distintas, entender los modelos actuales y generar nuevos, aprovechar los conceptos de negocio como tal y aplicar solo aquellos que respondan a las necesidades cambiantes de cada institución.

\section{Refencias}

DOS DE DOCE (2014). Nuevos modelos de negocio en la era digital, http://www.dosdoce.com/upload/ficheros/noticias/201409/modelos_de_negocio_pdf.pdf

LAUREN STYX (2021). Museumnext, How are museums using artificial intelligence, and is a AI the future of museums?, Museum Next.

MUSEUMS REPORT (2016): Digital Transformation in the Museum Industry. Axiell, Museum.

Archives and Librares Services, Reino Unido.

NMC Horizon Report Museum Edition (2016) Ecsite, A publication by the New Media Consortium. 